\title{
Childhood cognitive development in 22q11.2 deletion syndrome: case-control study
}

Samuel J. R. A. Chawner, Joanne L. Doherty, Hayley Moss, Maria Niarchou, James T. R. Walters, Michael J. Owen and Marianne B. M. van den Bree

\section{Background}

22q11.2 deletion syndrome (22q11.2DS) is associated with a high risk of childhood as well as adult psychiatric disorders, in particular schizophrenia. Childhood cognitive deterioration in 22q11.2DS has previously been reported, but only in studies lacking a control sample.

\section{Aims}

To compare cognitive trajectories in children with 22q11.2DS and unaffected control siblings.

\section{Method}

A longitudinal study of neurocognitive functioning (IQ, executive function, processing speed and attention) was conducted in children with 22q11.2DS ( $n=75$, mean age time $1\left(T_{1}\right) 9.9$, time $\left.2\left(T_{2}\right) 12.5\right)$ and control siblings $(n=33$, mean age $T_{1}$ 10.6, $T_{2}$ 13.4).

\section{Results}

Children with 22q11.2DS exhibited deficits in all cognitive domains. However, mean scores did not indicate deterioration.
When individual trajectories were examined, some participants showed significant decline over time, but the prevalence was similar for 22q11.2DS and control siblings. Findings are more likely to reflect normal developmental fluctuation than a 22q11.2DS-specific abnormality.

\section{Conclusions}

Childhood cognitive deterioration is not associated with 22q11.2DS. Contrary to previous suggestions, we believe it is premature to recommend repeated monitoring of cognitive function to identifying individual children with 22q11.2DS at high risk of developing schizophrenia.

\section{Declaration of interest}

None.

\section{Copyright and usage}

(c) The Royal College of Psychiatrists 2017. This is an open access article distributed under the terms of the Creative Commons Attribution (CC BY) licence.
According to the neurodevelopmental hypothesis, subtle cognitive deficits emerge years before adult schizophrenia becomes manifest. ${ }^{1,2}$ Pre-existing deficits have been documented in children who later develop schizophrenia, but their developmental course remains unclear. ${ }^{3}$ An important question is whether childhood cognitive deterioration is associated with schizophrenia development risk. When investigating whether the developmental course of cognitive domains represents cognitive deterioration or is more likely to reflect other patterns of cognitive development (Fig. 1), it is necessary to consider changes in raw scores (i.e. number of correct items on an IQ subtest) as well as agestandardised scores (i.e. IQ). The developmental deterioration hypothesis ${ }^{4,5}$ predicts an absolute loss of previously acquired absolute ability. In contrast, the developmental lag hypothesis ${ }^{6}$ predicts growth in absolute ability which, however, lags behind the general population rate. Both developmental deterioration and developmental lag result in the decline of age-standardised scores such as IQ and can only be distinguished using raw scores, with the first hypothesis predicting decline in raw score and the second increase in raw score (Fig. 1). The developmental deficit hypothesis on the other hand predicts a static deficit in age-adjusted ability, ${ }^{7}$ whereby impaired cognition manifests early in development, but the rate of cognitive development is the same as in typically developing individuals. Finally, developmental maturation is characterised by an initial deficit in absolute ability, which decreases with age (i.e. a 'catch-up' effect).

$22 \mathrm{q} 11.2$ deletion syndrome (22q11.2DS) is one of the strongest known risk factors for schizophrenia with a rate of $\sim 30 \%$ reported in adult patients. ${ }^{8}$ 22q11.2DS affects 1 in 4000 live births and the associated phenotype is highly variable including congenital abnormalities, developmental delay and high rates of childhood psychiatric disorders such as autism, attention-deficit hyperactivity disorder and anxiety. ${ }^{8,9}$ Studies of children with the deletion provide a unique opportunity to prospectively examine development before schizophrenia onset. Some, ${ }^{10-15}$ but not all, ${ }^{16,17}$ longitudinal studies of cognition in children with 22q11.2DS report evidence of verbal IQ (VIQ) decline. It remains unknown in 22q11.2DS whether cognitive decline represents developmental deterioration in absolute ability or a developmental lag in acquiring absolute ability as no 22q11.2DS study has been able to examine change in IQ subtest raw score across their whole cohort because the same IQ test version has not been administered at both time points and with all participants. Different Wechsler test versions have been used and although similar in design and psychometric properties, raw score performance is not comparable across different test versions. Developmental deterioration and lag are likely to be underpinned by different biological processes, with deterioration indicating a brain degenerative process, whereas a lag may indicate impaired brain developmental processes that manifest through adolescence. Distinguishing these processes is important to understanding the mechanisms underlying cognitive decline in 22q11.2DS.

Studies of cognitive development in 22q11.2DS have suffered other methodological limitations. First, only a few have included a control group. ${ }^{11,16-20} \mathrm{~A}$ control group is needed to account for extraneous methodological factors such as measurement error, regression to the mean, practice effects and the possibility that changes in performance over time represent normal developmental fluctuation. This important point is demonstrated by a study that found that improvement in cognitive performance following antipsychotic medication in patients with schizophrenia was because of a practice effect rather than medication, as this effect was found in healthy controls who were not taking medication. ${ }^{21}$ Second, studies of children with 22q11.2DS should take account of 


\begin{tabular}{|c|c|c|c|c|}
\hline $\begin{array}{c}\text { Model of } \\
\text { cognitive } \\
\text { development }\end{array}$ & Visualisation & $\begin{array}{l}\text { Controls } \\
\text { Affected } \\
\text { group }\end{array}$ & Description & Expectation for raw score \\
\hline $\begin{array}{l}\text { Developmental } \\
\text { deficit }\end{array}$ & 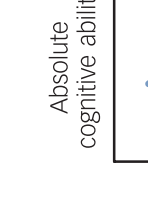 & & $\begin{array}{l}\text { A static cognitive } \\
\text { impairment that } \\
\text { emerges early in } \\
\text { development and } \\
\text { remains stable. }\end{array}$ & $\begin{array}{l}\text { 22q11.2DS and control raw scores } \\
\text { increase with age at the same rate. } \\
\text { No interaction between } \\
\text { deletion status and age. }\end{array}$ \\
\hline $\begin{array}{l}\text { Developmental } \\
\quad \text { lag }\end{array}$ & 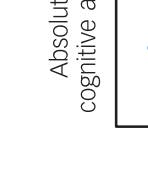 & & $\begin{array}{l}\text { Growth in absolute } \\
\text { ability, but growth } \\
\text { that lags behind that } \\
\text { in typical individuals. }\end{array}$ & $\begin{array}{l}\text { 22q11.2DS raw scores increase at } \\
\text { a slower rate than in controls. } \\
\text { Negative interaction between } \\
\text { deletion status and age. }\end{array}$ \\
\hline $\begin{array}{l}\text { Developmental } \\
\text { deterioration }\end{array}$ & 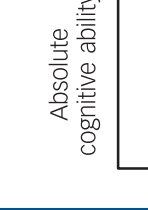 & & $\begin{array}{l}\text { Decline in absolute } \\
\text { ability. }\end{array}$ & $\begin{array}{l}\text { Raw scores decrease in 22q11.2DS } \\
\text { and increase in controls with age. } \\
\text { Negative interaction between } \\
\text { deletion status and age. }\end{array}$ \\
\hline $\begin{array}{l}\text { Developmental } \\
\text { maturation }\end{array}$ & 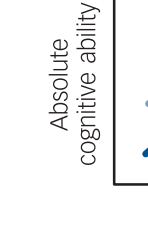 & & $\begin{array}{l}\text { Initial cognitive } \\
\text { impairment but } \\
\text { development catches } \\
\text { up with typical } \\
\text { individuals. }\end{array}$ & $\begin{array}{l}\text { Raw scores increase in } 22 \mathrm{q} 11.2 \mathrm{DS} \\
\text { at a faster rate than in controls. } \\
\text { Positive interaction between } \\
\text { deletion status and age. }\end{array}$ \\
\hline
\end{tabular}

Fig. 1 Descriptions of different models of cognitive development and expectations of raw scores for children with 22 q11.2 deletion syndrome (22q11.2DS) and control siblings.

individual trajectories as well as examining group trajectory, only a few previous studies have considered this. ${ }^{12,15}$ Adolescence is known to be a period when considerable changes occur in brain structure, function and cognitive development. ${ }^{22}$ Although IQ is highly stable at the group level in typically developing adolescents, ${ }^{23}$ there is also evidence of large intra-individual changes over time. ${ }^{24}$ Individual fluctuations may therefore average out at the group level. These findings indicate that considerable individual change, including possible cognitive deterioration may not be an uncommon occurrence in typically developing adolescents. The interpretation of the cognitive trajectory of 22q11.2DS can benefit from comparison with a typically developing sample. Third, as different aspects of cognition have been associated with schizophrenia risk, a focus solely on the developmental pathway of IQ would give an incomplete picture. Only a few longitudinal studies to date have, however, explored other neurocognitive domains in $22 \mathrm{q} 11.2 \mathrm{DS}^{16,20,25}$ and none has examined cognitive deterioration in neurocognitive domains other than IQ.

To date, only two studies, based on The Netherlands nationwide 22q11.2DS cohort, have specifically investigated cognitive deterioration. The authors examined IQ subtest raw scores in a subgroup of their cohort, between ages 7.5 and 9.5 years, ${ }^{12}$ and 9.5 and 15.3 years, ${ }^{15}$ and both studies reported that approximately a third exhibited deterioration. However, these studies lacked a control group, so it remains uncertain whether this phenomenon can be specifically attributed to the $22 \mathrm{q} 11.2$ deletion. These studies did not investigate other models of cognitive development (developmental lag, deficit, maturation).
The present study presents findings from the Cardiff University ECHO (Experiences of CHildren with cOpy number variants) study longitudinal cohort of children with 22q11.2DS. We address the limitations of previous longitudinal 22q11.2DS studies by including a comparison group of similar-aged, unaffected control siblings, and we administered the same IQ test version and neurocognitive battery to all participants at both waves. The goals of this study were to conduct: (a) a group-level analysis to characterise average cognitive trajectories in 22q11.2DS relative to control siblings and, in particular, to examine whether children with 22q11.2DS on average exhibit cognitive deterioration; (b) to classify trajectories of individuals to investigate if there is a subgroup of children with 22q11.2DS who exhibit cognitive deterioration and whether this differs from controls. Based on findings from The Netherlands nationwide 22q11.2DS cohort, we hypothesised that children with 22q11.2DS would exhibit cognitive deterioration compared with controls.

\section{Method}

\section{Participants}

The Cardiff ECHO study has assessed individuals with 22q11.2DS longitudinally through childhood and adolescence. In total, 75 of 102 children (73.5\%) with 22q11.2DS with baseline assessments were reassessed. The study also assesses control siblings closest in age to the index child, 33 of $49(67.3 \%)$ were reassessed. The mean age of the children with 22q11.2DS at time $1\left(T_{1}\right)$ was 9.9 
(s.d. $=2.4)$; time $2\left(T_{2}\right)=12.5$ (s.d. $\left.=2.3\right)$, and $44(59 \%)$ were male. Control siblings at $T_{1}$ were aged $10.6($ s.d. $=2.0) ; T_{2}=13.4$ (s.d. $=2.0)$, and $17(52 \%)$ were male. The gap between $T_{1}$ and $T_{2}$ was 2.7 (s.d. $=0.5$, range $2.0-4.0$ ) years. $T_{1}$ and $T_{2}$ age did not differ between the 22q11.2DS and control groups (independent $t$-test; $\left.T_{1}, P=0.150, T_{2}, P=0.060\right)$. Baseline and longitudinal recruitment is ongoing, the current study reports on participants recruited and assessed between March 2010 and February 2016. For both children with 22q11.2DS and control siblings reassessment was not associated with gender, baseline age and baseline IQ (online Table DS1).

At $T_{1}$ participants were recruited through National Health Service (NHS) medical genetics clinics in the UK, British 22q11.2DS charities Max Appeal and The 22Crew, and rare chromosomal disorder support group Unique. Children with 22q11.2DS were eligible if the 22q11.2 deletion was confirmed via medical genetics clinics and the Division of Psychological Medicine and Clinical Neurosciences laboratory. Inclusion for controls was based on being a biological sibling and not having a diagnosis of 22q11.2DS. Where multiple siblings were present in a family the child closest in age to the index child was invited to take part. All participating children were above the age of 6 years, because our cognitive measures would not be valid at younger ages. We did not exclude children with 22q11.2DS and control siblings based on physical or psychiatric phenotype. Psychiatric assessment details and diagnoses for participants can be found in the online Table DS2 and are consistent with those reported in previous studies. ${ }^{8,9}$ It is important to note that no individuals had developed psychotic disorder. Informed consent was gained from primary carers and participants. Protocols were approved by NHS Wales Research Ethics Committee.

\section{Assessments}

Cognitive test-retest data were available for 69 children with 22q11.2DS and 31 controls. Missingness was variable across cognitive measures (Fig. 2). Age-adjusted scores were derived and transformed to a $z$-score using norms available for each measure. IQ was assessed using the Wechsler Abbreviated Scale of Intelligence (WASI), ${ }^{26}$ from which full-scale IQ (FSIQ), VIQ and performance IQ (PIQ) were derived. These scores are already age-standardised and we also derived subtest age-adjusted and raw score performance.

Neurocognitive domains were assessed using the Wisconsin Card Sorting Test (WSCT) ${ }^{27}$ where number of perseverative errors measured the set-shifting ability aspect of executive function. The following metrics were derived from performance on CANTAB (Cambridge Neuropsychological Test Automated Battery) ${ }^{28}$ tests: number of errors on the spatial working memory (SWM) task measured executive function ability; number of problems solved on the Stockings of Cambridge (SOC) task measured spatial planning, another aspect of executive function; five-choice reaction time in milliseconds (RTI) measured processing speed; number of correct items on the match-to-sample task (MTS) task measured visual attention; and a score $0-1$ that represents the participant's ability to discriminate target stimuli from distractor stimuli on the rapid visual information processing task measured sustained attention. For MTS only raw scores were used because there are no CANTAB norms for this task.

\section{Statistical analysis}

\section{Aim 1: group-level analysis}

Cognitive trajectories by age for children with 22q11.2DS and controls were estimated using linear mixed models. The model included age, gender, deletion status, the interaction between age and deletion status and the interaction between gender and deletion status as fixed effects, familial clustering was controlled for by including family number as a random effect and repeated effects (to take account of collinearity, repeated $T_{1}$ and $T_{2}$ measures included age and cognitive scores) were also included. The magnitude of cognitive deficit ( $z$-score) was estimated in children with 22q11.2DS relative to controls, representing the average deficit across time points.

Analysis of raw scores defined the developmental trajectory of cognition in children with 22q11.2DS relative to controls. The effect of the deletion on raw score was tested (main effect of deletion status) as well as whether rate of change in raw score differed between children with 22q11.2DS and controls (interaction between age and deletion status on cognitive score). The direction of change in raw score was considered, a negative change indicated developmental deterioration. For FSIQ, VIQ and PIQ linear mixed models estimated the divergence score per year between children with 22q11.2DS and controls (interaction between deletion status and age on cognitive score). FSIQ, VIQ and PIQ do not have a raw score equivalent as they are composite scores of subtests with differing raw score scales. Findings were used to characterise cognitive development using the criteria described in Fig. 1.

\section{Aim 2: inter-individual analysis}

For aim 1, linear mixed-model analysis was conducted to examine deterioration at the group level, but we also wanted to investigate evidence of cognitive deterioration at the individual level. Thus, we explored whether cognitive deterioration occurred in a subgroup of children with 22q11.2DS and, if so, whether this proportion differed from control siblings. To examine this, we classified individuals based on change in raw score. For processing speed, SWM and set-shifting ability the sign of the change score was reversed so that a higher score reflected better performance for these tasks.

First, individuals were categorised based on whether they exhibited a negative change in raw score $(<0)$. Fisher's exact test was conducted to determine whether there were differences in the percentage of children showing cognitive decline between the two groups.

Second, we wanted to investigate whether a negative change in raw score represented reliable cognitive deterioration. If an individual decreased in cognitive raw score by only a couple of points, this could represent measurement error, i.e. chance fluctuation, rather than a real cognitive deterioration effect. To determine if raw score change was reliable, change scores were categorised using cut-offs based on reliable change indices (RCIs). ${ }^{29}$ These were calculated from the standard error of prediction $\quad\left(\mathrm{SEP}=\right.$ s.d. $\times\left(1-r^{2}{ }_{12}\right)^{1 / 2}$; where s.d. is standard deviation of the measure, and $r_{12}^{2}$ is the test-retest reliability coefficient of the measure, metrics gained from published values $^{26,27,30}$ ), RCI cut-off is plus or minus 1.96 SEP. Fisher's exact test was conducted to test whether deletion status was associated with reliable cognitive deterioration. For children with 22q11.2DS we also examined whether reliable cognitive deterioration was associated with meeting criteria for any psychiatric disorder using Fisher's exact test.

\section{Results}

Table 1 displays cognitive test scores for children with 22q11.2DS and controls at $T_{1}$ and $T_{2}$. 


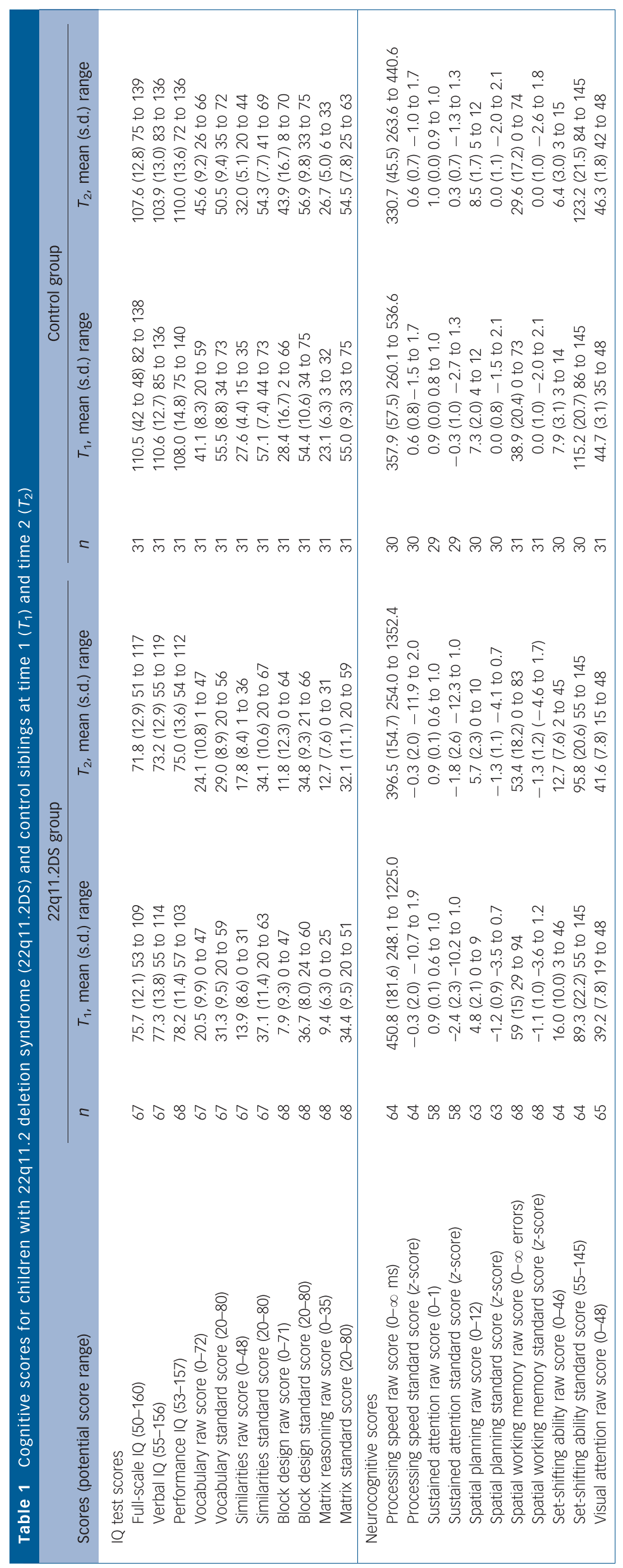


Aim 1: group-level analysis

Children with 22q11.2DS had significant deficits across all cognitive measures compared with controls. The magnitude of the deficit was domain specific, with SWM, spatial planning and processing speed relatively less impaired than other cognitive measures (Fig. 2). There was no evidence that development of any of the cognitive domains assessed fitted the model of developmental deterioration (online Fig. DS1).

Change over time in VIQ fitted a developmental deficit model as there was no evidence that the trajectories for children with 22q11.2DS and controls diverged (interaction $P=0.693$, online Fig. DS1(a)). This was the case for both VIQ subtests, vocabulary (interaction $P=0.915$ ) and similarities (interaction $P=0.314$ ). Change in PIQ over time, however, fitted a developmental lag model as children with 22q11.2DS had a worsening deficit of 2.1 (95\% CI 0.7-3.5) PIQ points per year relative to controls (interaction $P=0.004$ ) (Table 2, online Fig. DS1(a)). Examination of the subtests that comprise PIQ indicated this was driven by block design (interaction $P<0.001$ ) rather than matrix reasoning performance (interaction $P=0.185$ ) (Table 2). This appears be the result of a floor effect in individuals with 22q11.2DS at $T_{1}$ on the block design task (online Fig. DS2(b)), rather than a true developmental lag effect.
Children with 22q11.2DS showed a 1.0 ( $95 \%$ CI -0.6 to 2.4 ) decline in FSIQ per year compared with controls. However, there was no evidence for an interaction between deletion status and age on FSIQ $(P=0.218)$, representing a developmental deficit model (Table 2; Fig. DS1(a)). For processing speed (RTI) there was evidence of developmental maturation (interaction $P=0.018$; Table 2, online Fig. DS1(c)). Change over time for the remaining cognitive domains fitted a developmental deficit model as there was no evidence for divergence in cognitive trajectories between children with 22q11.2DS and controls (Table 2, online Fig. DS2(c)).

There was an effect of gender by deletion status (22q11.2DS, control) interaction $(P=0.003)$ on block design performance, however, this was driven by a gender difference in the control group (male-female 26.7, $P=0.037$ ) rather than in children with 22q11.2DS average ability; 22q11.2DS (male-female -4.7 , $P=0.285$ ). For the other cognitive domains, there was no effect of gender.

Online Fig. DS1 shows the trajectories of every participant, providing an indication of the inter-individual differences seen in both children with $22 \mathrm{q} 11.2 \mathrm{DS}$ as well as control siblings. For all cognitive measures, the percentage of children with 22q11.2DS who exhibited decline in cognitive performance raw score did not differ from controls (online Table DS4). Furthermore, the

$n$ (22q11.2DS, Controls)

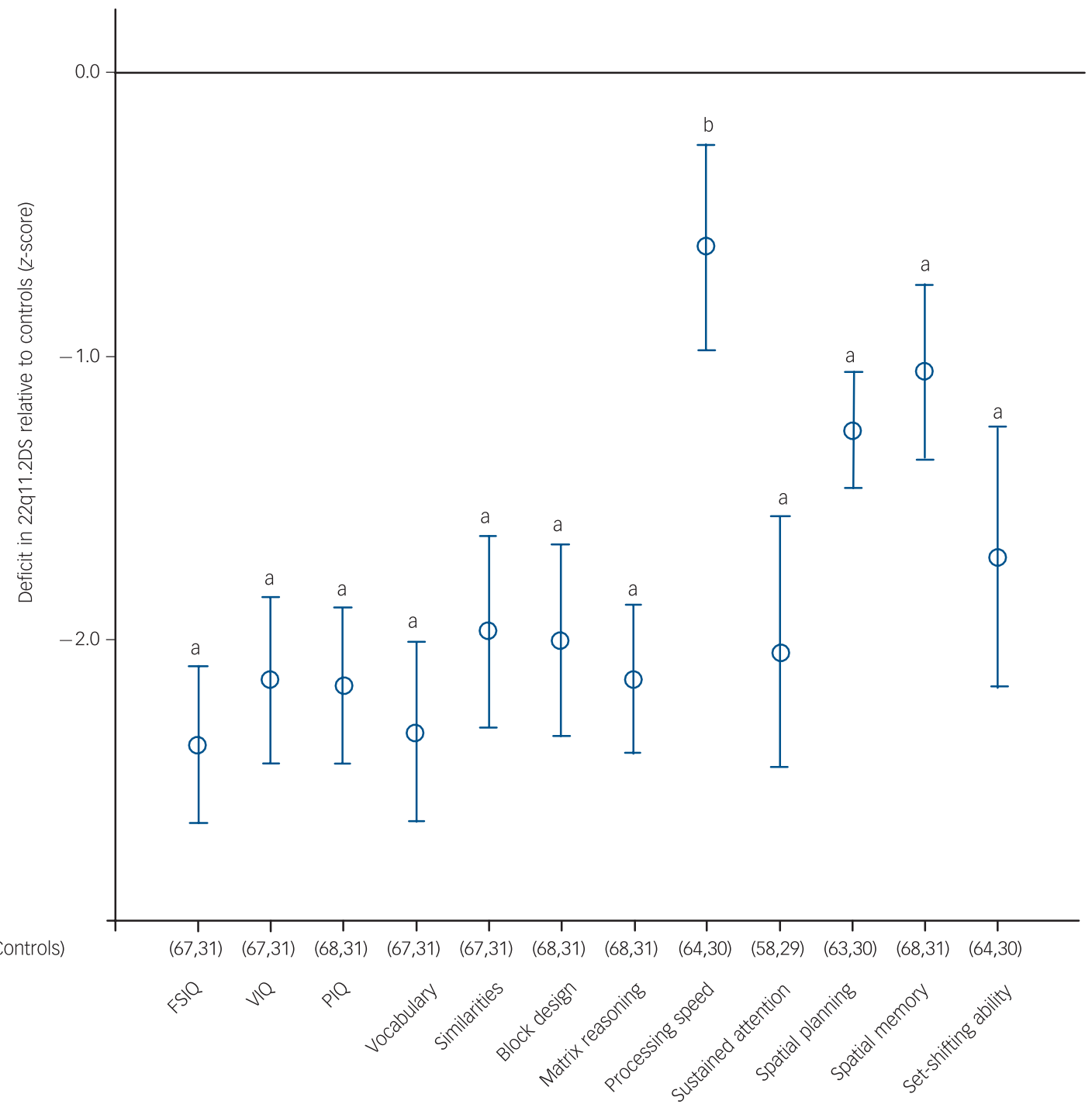

Fig. 2 Cognitive development in children with 22q11.2 deletion syndrome (22q11.2DS) relative to control siblings. 


\begin{tabular}{|c|c|c|c|c|c|c|}
\hline & $\begin{array}{l}2 \mathrm{q} 11.2 \mathrm{DS} \\
\text { group, } n\end{array}$ & $\begin{array}{l}\text { Control } \\
\text { group, } n\end{array}$ & $\begin{array}{c}\text { Interaction } \\
P\end{array}$ & $\begin{array}{l}2 \mathrm{q} 11.2 \mathrm{DS} \text { group change } \\
\text { relative to control group }\end{array}$ & $\begin{array}{l}\text { Raw score performance over } \\
\text { time in 2q11.2DS group }\end{array}$ & Model \\
\hline \multicolumn{7}{|l|}{ Standardised scores } \\
\hline Full-scale IQ & 67 & 31 & 0.218 & 1.0/year & Increased & Developmental deficit \\
\hline Verbal IQ & 67 & 31 & 0.693 & $0.4 /$ year & Increased & Developmental deficit \\
\hline Performance IQ & 68 & 31 & 0.004 & 2.1/year & Increased & Developmental lag \\
\hline \multicolumn{7}{|l|}{ Raw scores } \\
\hline Vocabulary & 67 & 31 & 0.915 & No relative change & Increased & Developmental deficit \\
\hline Similarities & 67 & 31 & 0.314 & No relative change & Increased & Developmental deficit \\
\hline Block design & 68 & 31 & $<0.001$ & Divergence & Increased & Developmental lag \\
\hline Matrix reasoning & 68 & 31 & 0.185 & No relative change & Increased & Developmental deficit \\
\hline Processing speed & 64 & 30 & 0.018 & Convergence & Increased & Developmental maturation \\
\hline Sustained attention & 58 & 29 & 0.304 & No relative change & Increased & Developmental deficit \\
\hline Spatial planning & 63 & 30 & 0.674 & No relative change & Increased & Developmental deficit \\
\hline spatial memory & 68 & 31 & 0.098 & No relative change & Increased & Developmental deficit \\
\hline Set-shifting ability & 64 & 30 & 0.244 & No relative change & Increased & Developmental deficit \\
\hline Visual attention & 65 & 31 & 0.191 & No relative change & Increased & Developmental deficit \\
\hline
\end{tabular}

percentage of children who exhibited reliable cognitive deterioration did not differ from controls. For the IQ subtests and set-shifting ability there were no children with 22q11.2DS who met the cut-off for reliable cognitive deterioration. In children with 22q11.2DS no relationship was found between deterioration in raw score and the presence of any psychiatric diagnosis at either $T_{1}$ and $T_{2}$, similarly there was no relation between reliable cognitive deterioration and the presence of any psychiatric disorder at $T_{1}$ or $T_{2}$ (data not presented).

\section{Discussion}

\section{Main findings and comparison with findings from other studies}

This is the first longitudinal study of cognition in 22q11.2DS that has compared different models of cognitive development. We are also the first study of cognitive deterioration in 22q11.2DS that has used a control group. This was possible because of the availability of a control sibling sample and the administration of identical cognitive measures at both time points. This methodology allowed us to explore whether cognitive decline represents a developmental lag or an absolute developmental deterioration. We found that cognitive deterioration is not a developmental feature specific to 22q11.2DS. Across all cognitive domains, we found no evidence that the group trajectory associated with 22q11.2DS represented developmental deterioration. This indicates that on average children with 22q11.2DS do continue to acquire ability and knowledge throughout childhood and adolescence. This is also consistent with findings in the Dunedin cohort, where children who later developed schizophrenia did not exhibit cognitive deterioration. ${ }^{31}$ The findings suggest that schizophrenia risk is not associated with neurodegenerative brain processes during childhood and adolescence. We also examined individual cognitive trajectories, and found that for IQ subtest performance as well as set-shifting ability none of the children with 22q11.2DS or controls met criteria for reliable cognitive deterioration. There were children with 22q11.2DS who exhibited reliable cognitive deterioration in processing speed, attention domains, spatial planning and SWM. However, our ability to compare children with 22q11.2DS with their siblings leads us to conclude that the reliable cognitive deterioration we found was not specific to children with 22q11.2DS, as the prevalence of reliable cognitive deterioration in children with 22q11.2DS did not differ from siblings. Large individual cognitive changes have been reported in adolescence in the typically developing population. $^{24}$ This suggests our findings in children with 22q11.2DS reflect developmental changes that are common to all adolescents.

Cognitive deterioration was not found to be related to the presence of psychiatric disorder, which is consistent with findings from The Netherland's cohort. ${ }^{12,15}$ To define reliable cognitive deterioration, we used cut-offs to determine if raw score changes were above and beyond change because of measurement error. This approach indicated that although some children with 22q11.2DS exhibit a decrease in raw score on IQ subtests, it was not at a greater magnitude than what can be attributed to measurement error. Some authors have recommended regular IQ testing for children with 22q11.2DS to identify children who exhibit cognitive deterioration and may therefore possibly be at risk of developing psychosis, ${ }^{32}$ however, our comparisons of children with and without the deletion indicate that the large fluctuations over time as well as the lack of reliable change may mean the results of such tests will be difficult to interpret. We found that children with 22q11.2DS have significant impairment across all cognitive domains, however, we do not find persuasive evidence that they show a decline in cognition. Because we used a case-control design we could establish that VIQ does not decline relative to control siblings, but that instead a static developmental deficit was present. Although our findings differ from previous 22q11.2DS studies, our findings show striking consistency with premorbid verbal cognitive development observed in individuals from the population-based Dunedin cohort who later developed schizophrenia. ${ }^{31}$ It should be noted that within both 22q11.2DS and control sibling groups there was VIQ decline, but as the magnitude was similar, we do not find relative decline in children with 22q11.2DS. This could indicate that our controls are not representative, however, this phenomenon of decline in verbal ability in controls is not uncommon within the schizophrenia literature. ${ }^{3}$ This emphasises the importance of a case-control design as this allows extraneous factors such as measurement error, regression to the mean and practice effects to be taken into account when interpreting cognitive change.

Children with 22q11.2DS also exhibited developmental deficits in various aspects of executive function and attention. 
We found that the magnitude of the cognitive deficits in children with 22q11.2DS was domain specific; deficits in spatial planning and SWM aspects of executive function and processing speed were relatively less impaired than IQ, attention and set-shifting ability. This is consistent with a cross-sectional study of 22q11.2DS that reported greater deficits in complex cognition and relative strengths in working memory and spatial processing. ${ }^{33}$ We found developmental maturation for processing speed, suggesting childhood deficits in this domain reduce through adolescence. This mirrors longitudinal findings from a typically developing cohort whereby children who develop psychotic experiences have initial deficits but subsequently 'catch-up' in processing speed ability. ${ }^{34}$ We found that PIQ appears to decline 2.1 PIQ points per year relative to controls, which would indicate a developmental lag. However, closer examination at subtest raw score performance suggests this is unlikely to represent true cognitive decline. The developmental lag in PIQ was driven only by a developmental lag in the block design subtest that appears to be the result of a floor effect. At $T_{1}$ many individuals in the 22q11.2DS group were scoring at the floor of the subtest, which artificially deflated the relative deficit between individuals with 22q11.2DS and controls at $T_{1}$, thus giving the impression of a developmental lag across time. This points to another advantage of the opportunity to examine raw cognitive scores. Previous longitudinal studies of cognition in both the schizophrenia and 22q11.2DS literature that report either cognitive decline/or developmental lags have not always examined raw scores and therefore would not have been able to identify such possible floor effects. Standard IQ measures may not be the most suitable for assessing longitudinal cognitive change in individuals with cognitive impairments who are more likely to perform at the floor of the cognitive range these measures assess. We believe it would be helpful if more studies would present information on variation in both individual as well as average trajectories (online Fig. DS1). Unlike previous studies ${ }^{12,35}$ we did not find any evidence of gender differences in cognitive development in children with 22q11.2DS.

Overall, our finding of developmental deficits and maturation in 22q11.2DS highlights that although children with 22q11.2DS have significant cognitive impairment, they acquire new knowledge and cognitive ability throughout childhood and adolescence at a rate that is not lower than in typically developing children. The presence of developmental deficits suggests that cognitive impairment in 22q11.2DS emerges very early in development, consistent with the neurodevelopmental hypothesis of schizophrenia. The cognitive changes captured by our study are not because of the development of psychosis as no individual met criteria for psychotic disorder at either time point.

\section{Limitations}

This study has limitations; the average time gap between the two waves of study was only 2.7 years, which may have been too short to detect cognitive deterioration. However, the time gap is similar to a previous 22q11.2DS study that detected cognitive change. ${ }^{12}$ However, it could be argued that a longer time gap between the cognitive assessments could have the effect of averaging out the effects of a series of large dynamic changes in cognitive development. In terms of variability in age at each time point, our cohort is similar to other longitudinal studies of 22q11.2DS, but not as narrow as The Netherland's cohort that investigated cognitive deterioration; ${ }^{12,15}$ our findings are therefore comparatively less developmentally specific than those reported in that cohort. Also, our findings cannot be extrapolated to older age ranges where cognitive deterioration associated with the development of psychotic disorder may occur. The domain-specific effects reported could also be because of differences in the psychometric properties of the cognitive measures. ${ }^{36}$ A limitation of our study, which represents a general problem within this field, is that scores at the low end are likely to be less valid. ${ }^{37}$ When norms are developed for cognitive tests there is often limited standardisation for individuals with low ability. Another important consideration is that we have assumed in analysis that raw scores lie on a linear scale whereby intervals between scores are equivalent, whereas this may arguably be less likely for low scores. Linear mixed-models analysis allowed us to examine cognitive trajectories from ages 6 to 17. However, a potential issue is that there may be cohort effects, in that younger children in the study may differ in extraneous factors from older individuals. Finally, there is a limit to what we can conclude from two waves of assessment; a third wave of assessment will be important to establish whether the reported trends remain present.

\section{Implications}

Utilisation of control groups is paramount when defining the phenotypic effect of a copy number variant; when we compared the children with 22q11.2DS with control siblings we found no evidence that cognitive deterioration is a developmental feature specific to $22 \mathrm{q} 11.2 \mathrm{DS}$ and we did not replicate previous findings of VIQ decline in 22q11.2DS. Although our conclusions contrast with previous 22q11.2DS studies, they are consistent with patterns observed in childhood in individuals with schizophrenia who do not carry the 22q11.2 deletion, supporting the conclusion that $22 \mathrm{q} 11.2 \mathrm{DS}$ is a representative model for characterising cognitive development associated with schizophrenia risk.

Developmental deficits and lags in cognitive development are a core feature of childhood neurodevelopmental disorders. ${ }^{38,39}$ Future work should disentangle whether patterns of cognitive development are specific to schizophrenia risk or are related to the known shared aetiology between schizophrenia and childhood neurodevelopmental disorders. It has been suggested that repeated monitoring of cognitive function to detect decline and thereby identifying those at risk of psychosis should be an integral part of managing young people with $22 \mathrm{q} 11.2 \mathrm{DS},{ }^{10}$ many of whom will be under the care of child and adolescent mental health services because of the high prevalence of childhood psychiatric disorders. ${ }^{9}$ Although cognitive assessment is important for informing the educational needs of a child with $22 \mathrm{q} 11.2 \mathrm{DS}^{40}$ and should be strongly encouraged, our findings suggest that it may be premature to recommend that such assessments are used to identify children at high risk of psychosis. We would not be comfortable nor feel confident in predicting which children from our cohort, who are yet to enter the period of psychosis development, are at greatest risk based on their cognitive change scores. We found no children with 22q11.2DS exhibited reliable deterioration in IQ subtest raw scores. Our findings suggest that more research is needed before cognitive assessment can be used to identify children with 22q11.2DS who are at high risk of developing psychosis.

Samuel J.R.A. Chawner, PhD, Joanne L. Doherty, MRCPsych, Hayley Moss, BSC, Maria Niarchou, PhD, James T.R. Walters, PhD, MRCPsych, Michael J. Owen, PhD, FRCPsych, Marianne B.M. van den Bree, PhD, Medical Research Council PhD, FRCPSych, Marianne B.M. van den Bree, PhD, Medical Research Counch
Centre for Neuropsychiatric Genetics and Genomics, Division of Psychological Medicine and Clinical Neurosciences, Cardiff University, Cardiff, UK

Correspondence: Marianne B.M. van den Bree, Medical Research Council Centre for Neuropsychiatric Genetics and Genomics, 2nd Floor Hadyn Ellis Building, Cardiff University, Maindy Road, Cathays, Cardiff CF24 4HQ, UK. Email: vandenbreemb@cardiff.ac.uk

First received 3 Nov 2016, final revision 31 May 2017, accepted 12 Jun 2017 


\section{Acknowledgements}

We are extremely grateful to all the families that participated in this study as well as to support charities Max Appeal, The $22 \mathrm{Crew}$ and Unique for their help and to the International 22q11.2 Brain and Behavior Consortium for their support. We would also like to thank the following people for contributing to the study: Jane Scourfield, PhD, MRCPsych, Aimee Davies, BSc, Sophie Andrews, MSc, Adam Cunningham, MSc, Hayley Moulding, BSC, Katie Price, BSC, Jade Bowyer, BSC, Laura Douglas, BSC, Hannah McCarthy, BSC, Natalie Meeson, BSC, Joshua Roberts, BSC and Jessica Townsend, BSC Medica Research Council Centre for Neuropsychiatric Genetics and Genomics, Division of Psychological Medicine and Clinical Neurosciences, Cardiff University.

\section{Funding}

This study was funded by the Baily Thomas Charitable Trust (2315/1), the Waterloo Foundation (WF918-1234), Wellcome Trust ISSF grant, the National Institute for Mental Health (5U01MH101724), Medical Research Council studentship (S.J.R.A.C. 1499282) Wellcome Trust Fellowships (M.N. 110222/Z/15/Z J.L.D. 505714), Wellcome Trust Strategic Award (503147), Medical Research Council Centre grant (G0801418) and Medical Research Council Programme grant (G0800509).

\section{References}

1 Weinberger DR. The pathogenesis of schizophrenia: a neurodevelopmental theory. In The Neurology of Schizophrenia (eds RA Nasrallah, DR Weinberger): 387-405. Elsevier, 1986.

2 Murray RM, Lewis SW. Is schizophrenia a neurodevelopmental disorder? BMJ 1987; 295: 681-682

3 Woodberry K, Giuliano A, Seidman L. Premorbid IQ in schizophrenia: a metaanalytic review. Am J Psychiatry 2008; 165: 579-87.

4 Jones PB, Done DJ. From birth to onset: a developmental perspective of schizophrenia in two national birth cohorts. In Neurodevelopment \& Adult Psychopathology (eds S Matcheri, S Keshavan, R Murray): 119-30. Cambridge University Press, 1997

5 Reichenberg A, Weiser M, Rapp MA, Rabinowitz J, Caspi A, Schmeidler J, et al. Elaboration on premorbid intellectual performance in schizophrenia premorbid intellectual decline and risk for schizophrenia. Arch Gen Psychiatry 2005; 62: 1297-304.

6 Fish B, Marcus J, Hans SL, Auerbach JG, Perdue S. Infants at risk for schizophrenia: sequelae of a genetic neurointegrative defect: a review and replication analysis of pandysmaturation in the Jerusalem Infant Development Study. Arch Gen Psychiatry 1992; 49: 221-35.

7 Weinberger DR. Implications of normal brain development for the pathogenesis of schizophrenia. Arch Gen Psychiatry 1987; 44: 660-9.

8 Schneider $\mathrm{M}$, Debbané $\mathrm{M}$, Bassett $\mathrm{A}$, Chow EW, Fung WL, van den Bree $\mathrm{M}$, et al. Psychiatric disorders from childhood to adulthood in 22q11.2 deletion syndrome: results from the international consortium on brain and behavior in 22q11.2 deletion syndrome. Am J Psychiatry 2014; 171: 627-39.

9 Niarchou M, Zammit S, van Goozen SHM, Thapar A, Tierling HM, Owen MJ et al. Psychopathology and cognition in children with 22q11.2 deletion syndrome. Br J Psychiatry 2014; 204: 46-54.

10 Vorstman JA, Breetvelt EJ, Duijff SN, Eliez S, Schneider M, Jalbrzikowski M, et al. Cognitive decline preceding the onset of psychosis in patients with 22q11.2 deletion syndrome. JAMA Psychiatry 2015; 72: 377-85.

11 Gothelf D, Eliez S, Thompson T, Hinard C, Penniman L, Feinstein C, et al. COMT genotype predicts longitudinal cognitive decline and psychosis in 22q11.2 deletion syndrome. Nat Neurosci 2005; 8: 1500-2.

12 Duijff SN, Klaassen PWJ, de Veye H, Beemer FA, Sinnema G, Vorstman JAS Cognitive development in children with 22q11.2 deletion syndrome. $\mathrm{Br} J$ Psychiatry 2012; 200: 462-8.

13 Antshel KM, Shprintzen R, Fremont W, Higgins AM, Faraone SV, Kates WR Cognitive and psychiatric predictors to psychosis in velocardiofacial syndrome: a 3-year follow-up study. J Am Acad Child Adolesc Psychiatry 2010; 49: 333-44.

14 Schneider $M$, Schaer $M$, Mutlu AK, Menghetti S, Glaser B, Debbané $M$, et al. Clinical and cognitive risk factors for psychotic symptoms in 22q11.2 deletion syndrome: a transversal and longitudinal approach. Eur Child Adolesc Psychiatry 2014; 23: 425-36.

15 Duijff SN, Klaassen PWJ, de Veye H, Beemer FA, Sinnema G, Vorstman JAS Cognitive and behavioral trajectories in 22q11DS from childhood into adolescence: a prospective 6-year follow-up study. Res Dev Disabil 2013; 34 2937-45.

16 Hooper SR, Curtiss K, Schoch K, Keshavan MS, Allen A, Shashi V. A longitudinal examination of the psychoeducational, neurocognitive, and psychiatric functioning in children with 22q11.2 deletion syndrome. Res Dev Disabil 2013; 34: 1758-69.

17 Schaer $\mathrm{M}$, Debbané $\mathrm{M}$, Cuadra MB, Ottet MC, Glaser B, Thiran JP, et al. Deviant trajectories of cortical maturation in 22q11.2 deletion syndrome
(22q11DS): a cross-sectional and longitudinal study. Schizophr Res 2009; 115 $182-90$

18 Gothelf D, Penniman L, Gu E, Eliez S, Reiss AL. Developmental trajectories of brain structure in adolescents with 22q11.2 deletion syndrome: a longitudinal study. Schizophr Res 2007; 96: 72-81.

19 Antshel KM, Shprintzen R, Fremont W, Higgins AM, Faraone SV, Kates WR. Cognitive and psychiatric predictors to psychosis in velocardiofacial syndrome: a 3-year follow-up study. J Am Acad Child Adolesc Psychiatry 2010; 49: 333-44.

20 Antshel KM, Fremont W, Ramanathan S, Kates WR. Predicting cognition and psychosis in young adults with 22q11.2 deletion syndrome. Schizophr Bull 2017; 43: 833-42.

21 Goldberg TE, Goldman RS, Burdick KE, Malhotra AK, Lencz T, Patel RC, et al. Cognitive improvement after treatment with second-generation antipsychotic medications in first-episode schizophrenia: is it a practice effect? Arch Gen Psychiatry 2007; 64: 1115-22.

22 Blakemore SJ, Choudhury S. Development of the adolescent brain implications for executive function and social cognition. J Child Psychol Psychiatry 2006; 47: 296-312.

23 Van Soelen IL, Brouwer RM, Van Leeuwen M, Kahn RS, Pol HEH, Boomsma DI. Heritability of verbal and performance intelligence in a pediatric longitudinal sample. Twin Res Human Genet 2011; 14: 119-28.

24 Ramsden S, Richardson FM, Josse G, Thomas MSC, Ellis C, Shakeshaft C, et al. Verbal and non-verbal intelligence changes in the teenage brain. Nature 2011; 479: 113-6.

25 Maeder J, Schneider M, Bostelmann M, Debbané M, Glaser B, Menghetti S, et al. Developmental trajectories of executive functions in 22q11.2 deletion syndrome. J Neurodev Disord 2016; 8: 1.

26 Wechsler D. Manual for the Wechsler Abbreviated Intelligence Scale (WASI). Pearson, 1999.

27 Heaton R, Chelune G, Talley J, Kay G, Curtiss G. Wisconsin Card Sorting Test Manual Revised and Expanded. Psychological Assessment Resources, 1993.

28 Cambridge Cognition. CANTAB Eclipse Version 3. Cambridge Cognition, 2006.

29 Lineweaver TT, Chelune GJ. Use of the WAIS-III and WMS-III in the context of serial assessments: interpreting reliable and meaningful change. In Clinical Interpretation of the WAIS-III and WMS-III (eds DS Tulsky, DH Saklofske, RK Heaton, et al): 303-37. Elsevier, 2003.

30 Harrison J, Iddon J, Stow I, Blackwell A, Jefferies M, Shah $\mathrm{P}$. Neuropsychological Test Automated Battery (CANTAB): Test-Retest Reliability Characteristics. CANTAB, 2006.

31 Reichenberg A, Caspi A, Harrington H, Houts R, Keefe RSE, Murray RM, et al Static and dynamic cognitive deficits in childhood preceding adult schizophrenia: a 30-year study. Am J Psychiatry 2010; 167: 160-9.

32 Swillen A, McDonald-McGinn D. Developmental trajectories in 22q11.2 deletion syndrome. Am J Med Genet C Semin Med Genet 2015; 169: 172-81.

33 Gur RE, Yi JJ, McDonald-McGinn DM, Tang SX, Calkins ME, Whinna D, et al. Neurocognitive development in 22q11.2 deletion syndrome: comparison with youth having developmental delay and medical comorbidities. Mol Psychiatry 2014; 19: 1205-11.

34 Niarchou M, Zammit S, Walters J, Lewis G, Owen MJ, van den Bree MB. Defective processing speed and nonclinical psychotic experiences in children: longitudinal analyses in a large birth cohort. Am J Psychiatry 2013; 170: $550-7$.

35 Antshel KM, AbdulSabur N, Roizen N, Fremont W, Kates WR. Sex differences in cognitive functioning in velocardiofacial syndrome (VCFS). Devel Neuropsychol 2005; 28: 849-69.

36 Chapman $\sqcup$, Chapman JP. Problems in the measurement of cognitive deficits. Psychol Bull 1973; 79: 380.

37 Hessl D, Nguyen DV, Green C, Chavez A, Tassone F, Hagerman RJ, et al. A solution to limitations of cognitive testing in children with intellectual disabilities: the case of fragile $X$ syndrome. J Neurodevelop Dis 2008; 1 : 33-45.

38 Coghill D, Hayward D, Rhodes S, Grimmer C, Matthews K. A longitudinal examination of neuropsychological and clinical functioning in boys with attention deficit hyperactivity disorder (ADHD): improvements in executive functioning do not explain clinical improvement. Psychol Med 2014; 44: 1087-99.

39 Fisch GS, Simensen R, Tarleton J, Chalifoux M, Holden JJ, Carpenter N, et al. Longitudinal study of cognitive abilities and adaptive behavior levels in fragile X males: a prospective multicenter analysis. Am J Med Genet 1996; 64: 356-61.

40 Bassett AS, McDonald-McGinn DM, Devriendt K, Digilio MC, Goldenberg P, Habel A, et al. Practical guidelines for managing patients with 22q11.2 deletion syndrome. J Pediatr 2011; 159: 332-9. 